\title{
Cardiac CT image segmentation
}

\author{
XU Zihang ${ }^{1, a}$ JIANG Rui ${ }^{2, b}$ \\ ${ }^{1}$ Faculty of Information Technology, Macau University of Science and Technology, Macau, China \\ ${ }^{2}$ Faculty of Information Technology, Macau University of Science and Technology, Macau, China \\ xzh_58@163.com ${ }^{a} 1458952792 @ q q . c o m{ }^{b}$
}

Keywords: CT image segmentation,Active contour,Contrast enhancement

\begin{abstract}
Image segmentation is an important technology for computer-aided diagnosis. Purpose of medical image segmentation is to separate the images' regions with special meanings and to make the segmentation as close as possible to the anatomical structure so as to provide a reliable foundation for clinical diagnosis and treatment and also for pathological study. Due to the complexity of human anatomy, the irregularity of organ and tissue shapes as well as the diversity between individuals, general medical image segmentation methods cannot be directly applied to achieve the ideal segmentation effect. Besides, medical images have different features which cannot endow segmentation methods with universality. Thus, a more targeted medical image segmentation method is needed. This paper is targeted at studying an effective method for segmenting cardiac images, namely, the segmentation of coronary arteries and the segmentation of ventricle and atrium (VA). According to the characteristics and special needs of medical images, a new method for cardiac image segmentation was proposed. This method combines contrast enhancement, threshold segmentation, morphological algorithm and level set-based active contour model. Furthermore, this method improves its own problems to ultimately achieve the algorithm in MATLAB platforms, obtaining a better segmentation result.
\end{abstract}

\section{Introduction}

Heart disease is one of the major diseases that harm human health [1]. Heart is a solid parenchymatous organ and the locations of its internal lesions can only be known by referring to imaging data. Image segmentation thus becomes the key to CT diagnosis of heart disease, thereby enabling the accuracy improvement of CT cardiac segmentation to become an important research topic [2]. Aiming at solving the issue of segmenting cardiac images, a lot of researches have been done by domestic and foreign scholars and some achievements have already been made [3-5].

In the segmentation of cardiac CT image, due to the low noise and the low contrast between background and targets, has always been a difficulty in the area of image segmentation. Classical image segmentation mainly adopts threshold method, boundary-based method and region-based method etc. The inherent defects of these methods make it difficult to extract targeted regions directly from high-noise and low-contrast cardiac images. In recent years, the active contour mode [6] has been widely used in areas including boundary detection [6], shape modeling [7-8], image segmentation [9-10] and motion capturing [11] by virtue of its accuracy, automaticity and its continuity of the final segmentation curve [6].

This paper, based on cardiac images' characteristics of strong noise and low contrast as well as prior knowledge of the shapes of ventricle and atrium, proposed a segmentation method based on the high contrast, Otsu, and morphological operations of dynamic histogram homogenization as well as based on region-based $\mathrm{C}-\mathrm{V}$ model. By a combination of pre-segmentation and fine segmentation, the contours of VA were successfully extracted. 


\section{Active Contour Model}

Parametric contour model, also known as "snake" model [6], is an explicit expression of the parametric curve under the Lagrangian coordinate system. It extracts contours by minimizing extremum value's energy functional at the object boundaries. For the parametric curve X (s) = (x (s), $y(s)), s \in[0,1]$, its objective functional is shown in the following formula:

$$
\mathrm{E}=\int_{0}^{1}\left\{\frac{1}{2}\left[\alpha\left|\mathrm{X}^{\prime}\right|(\mathrm{s})^{2}+\beta\left|\mathrm{x}^{\prime \prime}(\mathrm{s})\right|^{2}\right]+\mathrm{E}_{\text {ext }}(\mathrm{X}(\mathrm{s}))\right\} \mathrm{ds}(1)
$$

The first two are the elastic term and rigid term. They keep the curve smooth during evolution. The third is the image data-based external energy term. It drives the evolution curve to the boundary of an object. Small capture range and impossibility in handling the issue of topological changes in the process of evolution is the bottleneck for the method's practical application. In view of the deficiency of parametric contour model, researchers proposed a geometric contour model [20-21] based on curve evolution theory [15-17] and the level set method [18-19]. The main idea is to express the evolution curve $\gamma(\mathrm{t})$ by zero level set function $\varphi(\mathrm{x} \mathrm{y} \mathrm{t})$ in higher one-dimensional space. Since the curve is expressed implicitly, it can thus rightly deal with topological changes. The partial differential evolution equation of the geometric contour model is

$$
\frac{\partial \mathrm{u}}{\partial \mathrm{t}}=-\mathrm{F}|\nabla \mathrm{u}|
$$

Driven by the velocity function F, the curve converges to the boundary of the object.

\section{Region-based C-V Model}

The gradient-based level set method requires that the initial contour be located near the object. Otherwise, the evolution curve may converge to a local optimum. Based on the curve evolution theory, Mumford-Shah functional [22] and the level set theory, the literature [23] proposed the region-based C-V model. The convergence criterion is no longer based on local statistics - gradient, and the model will not be disturbed by the issue of local optimization. The evolution equation is as follows:

$$
\frac{\partial \mathrm{u}}{\partial \mathrm{t}}=\delta_{\varepsilon}(\mathrm{u}) \quad\left(\mu \operatorname{div}\left(\frac{\nabla \mathrm{u}}{|\nabla \mathrm{u}|}\right)-\mathrm{v}-\lambda_{1}\left(\mathrm{u}_{0}-\mathrm{c}_{1}\right)^{2}+\lambda_{2}\left(\mathrm{u}_{0}-\mathrm{c}_{2}\right)^{2}\right)
$$

The first term is the length adjustment term. When $\mu$ is a relatively large value, filter out noise points and other small-sized objects. The second term is the boundary term of the internal area of curvature. It accelerates the evolution speed of the curve. The last two are the average statistics of some measurements inside and outside the curve.

Due to the position independence of the model's initial contour, the initial evolution curve can be set at a random position in the image and the final curves will all converge to the optimum location. In the evolutionary process, the evolution curve is not always required to be kept as the symbolic distance function.

A relatively large time step can be selected and the convergence rate will be faster.

\section{Summary}

Specific experimental steps are as follows: (1) Enhance the contrast of images through dynamic histogram homogenization. (2) Design the selection procedures including the selection of rectangular regions of ventricle and atrium, and the areas outside selected regions whose pixel assignments were zero. (3) Select the appropriate threshold value to impose binarization segmentation on the targeted region and make "open" operation on the binary image. (4) Extract, with the help of C-V model, the contours of ventricle and atrium inside the region. According to the relative size of ventricle, atrium and the noise, select the appropriate value of $\mu$ to filter noise and obtain the object contour. 


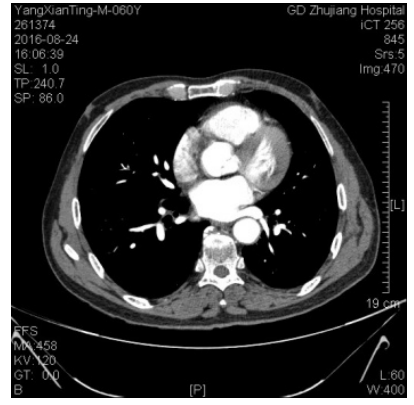

(a) original image

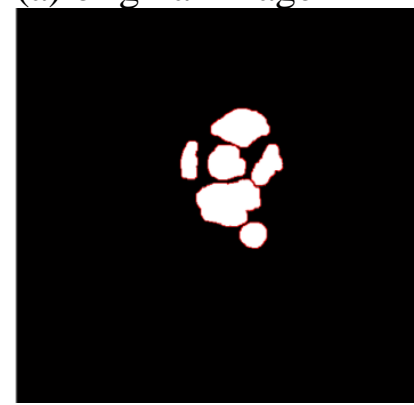

(d)C-V segmentation

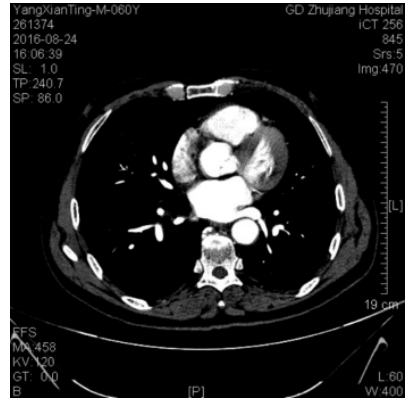

(b) Enhanced image

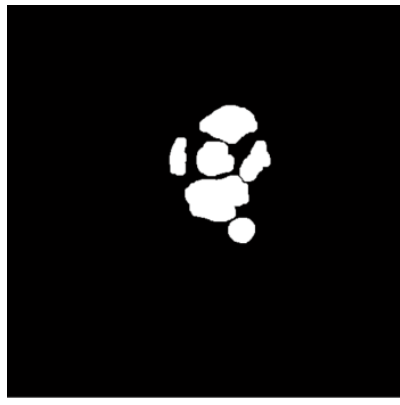

(c)Region of interest

Fig. 1 Cardiac CT image segmentation process

\section{References}

[1]Chen Xiaojun, Fu Zengliang, Ming, et al. Cardiac CT image segmentation method [J]. juice counting machine Engineering, 2009, 35 (12): 189-191.

[2] Karthikeyan C, Ramadoss B, Baskar S.Segmentation algorithm for CT images using morphological operation and artificial neural network[J].International Journal of Signal Processing, Image Processing and Pattern Recognition, 2012, 5 (2) : 115-122.

[3] Korea, Yang Shuyuan, Xia Jie, et al. A new segmentation of Watershed image based on mark Algorithm [J]. Journal of electronics, 2006, 34 (11): 2018-2023.

[4] Jiang Lin, Zhang Jianwei Li, Li Deyu, et al. Ultrasonic medicine based on morphological reconstruction Image filtering method [J]. Journal of Biomedical Engineering, 2007, 24 (3):

[5] Shang gorgeous. Medical CT image filtering method based on morphological reconstruction operation [J].China stereo vision and image analysis, 2011, 16 (1): 103-107.

[6] Kass M, Witkin A, Terzopoulos D. Snakes-active Contour Models[J]. International Journal of Computer Vision, 1987, 1(4): 321-331.

[7] Terzopoulos D, Fleischer K. Deformable Models[J]. Vis. Comput., 1988, 4(6): 306-331.

[8] McInerney T, Terzopoulos D. A Dynamic Finite Element Surface

Model for Segmentation and Tracking in Multidimensional Medical Image with Application to Cardiac 4D Image Analysis[J]. Comput. Med. Imag. Graph., 1995, 19(12): 69-83.

[9] Leymarie F, Levine M D. Tracking Deformable Objects in the Plane Using an Active Contour Model[J]. IEEE Trans. Pattern Anal. Machine Intell., 1993, 15(6): 617-634.

[10] Durikovic R, Kaneda K, Yamashita H. Dynamic Contour: A Texture Approach and Contour Operations[J]. Vis. Comput., 1995, 11(11): 277-289. 
[11] Terzopoulos D, Szeliski R. Tracking with Kalman Snakes[Z]. Active Vision, 1992.

[12] Yang, Wu Qiyao. Medical image technology and medical image segmentation [J]. world.2000, 6 (4):24-28.

[13] Chan T F, Vese L. Active Contours Without Edges[J].IEEE Trans. On Image Processing. 2001, 10(2):266-272.

[14] Bresson X, Esedoglu S, Vandergheynst P, et al. Fast Global Minimization of the Active Contour / Snake Model [J]. Journal of Mathematical Imaging and Vision. 2007,

[15] Sapiro G, Tannenbaum A. Affine Invariant Scale-space[J]. Int’l Journal Comp. Vis., 1993, 11(1): 25-44.

[16] Kimia B B, Tannenbaum A R, Zucker S W. Shapes, Shocks, and Deformations I: The Components of Two-dimensional Shape and the Reaction-diffusion Space[J]. Int'l Journal Comp. Vis., 1995, 15(3): 189-224.

[17] Alvarez L, Guichard F, Lions P L, et al. Axioms and Fundamental Equations of Image Processing[J]. Archive for Rational Mechanics and Analysis, 1993, 123(3): 199-257.

[18] Osher S, Sethian J A. Fronts Propagating with Curvature-dependent Speed: Algorithms Based on Hamilton-Jacobi Formulations[J]. Journal Computational Physics, 1988, 79(1): 12-49.

[19] Sethian J A. Level Set Methods and Fast Marching Methods: Evolving Interfaces in Computational Geometry, Fluid Mechanics, Computer Vision, and Material Science[M]. 2nd ed. Cambridge, UK: Cambridge University Press, 1999.

[20] Caselles V, Catte F, Coll T, et al. A Geometric Model for Active Contours[J]. Numer. Math., 1993, 66(1): 1-31.

[21] Caselles V, Kimmel R, Sapiro G. Geodesic Active Contours[J]. Int’l Journal Comp. Vis., 1997, 22(1): 61-79.

[22] Mumford D, Shah J. Optimal Approximation by Piecewise Smoot Functions and Associated Variational Problems[J]. Commun. Pure Appl. Math, 1989, (42): 577-685.

[23] Chan T F, Vese L. Active Contours Without Edges[J]. IEEE Trans. on Image Processing, 2001, 10(2): 266-272 